\title{
Efeito da prática de ioga na qualidade de vida de idosos
}

\author{
Fernanda Dias Massierer, ${ }^{1}$ Juliana Ludwig Justo, ${ }^{2}$ Adriana Marques Toigo ${ }^{3}$
}

\section{Resumo}

O envelhecimento é um fenômeno natural e fisiológico dos seres vivos, muitas vezes associado a pontos negativos, como degenerações, atrofias, limitações e dores. A ioga, muitas vezes, é usada como uma forma terapêutica que auxilia em recuperações e na manutenção da saúde e vem sendo uma boa ajuda para melhorar a qualidade de vida de idosos, minimizando os fatores negativos do envelhecimento. O objetivo deste estudo foi verificar se houve melhoras na qualidade de vida de idosos por meio da prática de ioga. Este estudo, caracterizado como descritivo-comparativo, foi composto por uma amostra final de 44 idosos entre 65 e 80 anos de idade, divididos em grupo experimental e grupo controle. Todos os idosos foram submetidos aos questionários Whoqol-old e Whoqol-bref para avaliação da qualidade de vida, e o grupo experimental foi submetido a aulas de ioga duas vezes por semana durante 10 semanas. Foram apresentadas diferenças estatisticamente significativas nas facetas Habilidades Sensoriais; Participação Social; Atividades Passadas, Presentes e Futuras; e Intimidade. Apesar de as outras facetas não apresentarem o mesmo resultado, houve um pequeno aumento nas variáveis analisadas.

Palavras-chave: Idosos. Qualidade de Vida. loga.

\section{Introdução}

A atividade física regular traz benefícios para o corpo e a mente de seres humanos. Acarreta o aumento de tônus muscular, o ganho de massa óssea; a diminuição dos percentuais de colesterol e glicose, dos índices de estresse, trazendo normalização do peso corporal (ANDERSON et al., 1998). Esses fatores contribuem para a melhora da qualidade de vida, especialmente em pessoas idosas, pois, segundo Mantovani (2013), pessoas acima dos 50 anos de idade representam a população mais sedentária entre os adultos, causando, assim, um envelhecimento inativo, no qual justamente a prática de atividades físicas significa

1 Bacharel em Educação Física pelo Centro Universitário La Salle. E-mail: femassierer@gmail.com

2 Licenciada em Educação Física pela Universidade Feevale. Mestre em Educação pelo Centro Universitário La Salle. Docente nos cursos de Licenciatura e Bacharelado em Educação Física do Centro Universitário La Salle.E-mail: jujusto@unilasalle.edu.br

3 Licenciada em Educação Física pela Universidade Federal do Rio Grande do Sul (Ufrgs). Mestre em Ciências do Movimento pela mesma instituição. Doutora em Ensino de Ciências pela Universidade de Burgos, Espanha. Docente nos cursos de Licenciatura e Bacharelado em Educação Física do Centro Universitário La Salle. Endereço para correspondência: Rua Prof. Ulisses Cabral, 1300/701, bl. 1, Porto Alegre, RS, 91330-520. E-mail: adrytoigo@terra.com.br

$\rightarrow$ http://dx.doi.org/10.5335/rbceh.v13i2.6120

Recebido em: 20.06.2016. Aceito em: 03.07.2017. 
manutenção da saúde e da qualidade da vida diária.

A qualidade de vida pode ser definida por conta de faixas etárias específicas de comunidades ou sociedades. É uma combinação de vários fatores que moldam e diferenciam o cotidiano do ser humano, como: estado de saúde; longevidade; satisfação no trabalho; salário; lazer; relações familiares; disposição; prazer e espiritualidade. Assim sendo, a qualidade de vida está diretamente relacionada com estilo e condições de vida. Nesse contexto, estão incluídos também hábitos, como tabagismo, alcoolismo, preferências alimentares, sedentarismo, entre outros (MANTOVANI, 2013), que podem afetá-la negativamente.

A ioga é uma prática conhecida há, pelo menos, cinco mil anos, com origem onde hoje é a Índia e o Paquistão (AQUINI, 2010). Existem várias técnicas utilizadas na ioga para fins específicos. Entre as principais, destacam-se os asanas (técnicas corporais), os pranayamas (técnicas respiratórias), os mantras (técnicas de vocalizações sonoras), os mudras (técnicas de postura de mãos e dedos), o yoganidra (técnica de descontração), o samyama (técnica de concentração) e o samadhi (técnica de meditação) (SOUZA; LISBOA, 2008).

São apresentados estudos em centros de pesquisas do mundo inteiro que abordam a ioga como uma forma terapêutica, a qual vem auxiliando nas recuperações e na manutenção da saúde (AQUINI, 2010).

De acordo com projeções do Fundo de Populações das Nações Unidas
(2016), "uma em cada nove pessoas no mundo tem 60 anos ou mais [de idade], e estima-se um crescimento para um em cada cinco por volta de 2050". Segundo o Instituto Brasileiro de Geografia e Estatística (IBGE), os idosos somam 23,5 milhões de brasileiros. Na última pesquisa divulgada, entre 2009 e 2011 , o grupo aumentou $7,6 \%$, ou seja, mais de 1,8 milhão de pessoas (BRASIL, 2002).

O envelhecimento é um fenômeno natural e fisiológico. Durante esse processo progressivo e irreversível, ocorrem alterações morfológicas e fisiológicas na estrutura corporal. O corpo é submetido a várias alterações, muitas vezes voltadas aos pontos negativos, como degenerações, atrofias, limitações, dores, paralelamente com a perda de qualidade de vida em seu dia a dia. Todas essas restrições acabam, por algum momento, afastando a pessoa idosa da sociedade (SOUZA; LISBOA, 2008).

De acordo com Iyengar (2011), o corpo envelhece e, se não houver movimentação, não haverá adequada irrigação sanguínea nele. Os asanas (posturas) permitem melhor circulação sanguínea nas extremidades e nas regiões mais profundas do corpo, possibilitando que as células se mantenham saudáveis.

A prática da ioga contribui na melhora do equilíbrio, da coordenação, dos níveis de força e da flexibilidade. Como consequência, a autonomia funcional, a autoconfiança e a prevenção contra quedas refletem positivamente no desempenho das atividades diárias e na qualidade de vida dos idosos (GONÇALVES, et al., 2010). 
Segundo Iyengar (2001), a ioga é considerada uma ciência que lida com a evolução da humanidade (entre aspectos do ser, da saúde física e da autorrealização). Presumindo que a ioga significa união, essa união vem de corpo e mente; fazendo com que essa prática cultive atitudes equilibradas da vida diária e forneça habilidades para a execução das ações.

A ioga vem mostrando boa aceitação por idosos. Além disso, sua prática ajuda na melhora de seus problemas de saúde, como: mudanças de humor, distúrbios do sono, fadiga, sintomas de estresse, queixas somáticas. Os praticantes relatam, também, melhoras em seu sistema imunológico, na flexibilidade, na autonomia funcional e no sistema metabólico, fatores que contribuem para a qualidade de vida de idosos (DIBENEDETTO et al., 2005).

O objetivo deste estudo foi verificar se houve diferença nas variáveis de qualidade de vida de idosos que participaram de um programa de ioga. Além disso, também se buscou comparar as mesmas variáveis de qualidade de vida (Habilidades Sensoriais; Autonomia; Atividades Passadas, Presentes e Futuras; Participação Social; Morte e Morrer; e Intimidade) entre o grupo de idosos que participaram de um programa de ioga e um grupo de idosos sedentários.

\section{Materiais e métodos}

Este estudo, caracterizado como descritivo-comparativo, foi aprovado pelo Comitê de Ética em Pesquisa com Seres
Humanos do Centro Universitário La Salle, Canoas, Brasil, em setembro de 2014 (protocolo de aprovação no 781.074). O campo de estudo foi o Centro Universitário La Salle (Unilasalle), localizado no município de Canoas, RS. O público investigado foi composto por idosos de ambos os sexos, com idades entre 65 e 80 anos, regularmente matriculados, em agosto de 2014, na Universidade Aberta da Terceira Idade (Unati), sediada no Unilasalle.

Foi utilizado o teste de Kolmogorov-Smirnov (K-S) para verificar a normalidade dos dados no grupo de praticantes de ioga e observou-se que somente as facetas do questionário Whoqol-old $\mathrm{Ha}$ bilidades Sensoriais e Autonomia, bem como do questionário Whoqol-bref, nos domínios Físico e Meio Ambiente, apresentaram distribuição normal para um intervalo de confiança de $95 \%$. Por essa razão, essas variáveis foram analisadas pelo teste $t$ de Student para amostras pareadas, e as demais, por não apresentarem distribuição normal, foram avaliadas pelo teste de Wilcoxon, análogo não paramétrico, para analisar diferenças entre duas condições no mesmo grupo de sujeitos em situação de pré-teste e pós-teste (PESTANA; GAGEIRO, 2003).

Foi novamente utilizado o teste de Kolmogorov-Smirnov (K-S), para verificar a normalidade dos dados em ambos os grupos, e observou-se que somente a faceta Participação Social do questionário Whoqol-old bem como o domínio Físico do questionário Whoqol-bref apresentaram distribuição normal para um intervalo de confiança de 95\%. Por essa 
razão, essas variáveis foram analisadas pelo teste $t$ de Student para amostras independentes, e as demais, por não apresentarem distribuição normal, foram avaliadas pelo teste de Mann-Whitney, análogo não paramétrico, para analisar diferenças entre dois grupos de casos quando há violação da normalidade (PESTANA; GAGEIRO, 2003).

Os critérios de exclusão para a participação no estudo incluíram idosos que já praticavam ioga dentro do período anterior a um ano até a data do início do estudo ou praticavam outros exercícios físicos concomitantemente; que não apresentaram atestado médico liberando a prática de exercício físico; ou, ainda, não tinham o mínimo de $75 \%$ de frequência nas aulas e/ou não faltavam duas aulas consecutivamente.

Todos os participantes da pesquisa assinaram o Termo de Consentimento Livre e Esclarecido (TCLE), atendendo a todas as exigências da legislação brasileira sobre experimentos com seres humanos.

A amostra final foi composta por 44 participantes (4 homens e 40 mulheres) com média de idade de 71,5 $\pm 75,3$ anos.

As aulas de ioga ocorreram duas vezes por semana, durante 10 semanas, em uma sala com piso de tatame, onde todos os participantes do grupo experimental (GE) se posicionavam aleatoriamente à frente da professora. A professora tinha vivência com ioga desde a infância, era formada em Hatha Yoga há 1 ano e estudou de Iyengar Yoga por mais de 3 anos. Cada sessão teve tempo de duração média de 45 minutos, utilizando uma sequência de asanas, tendo como referência os asanas propostos por Iyengar (2001).

Entre os asanas propostos, estavam: Tadasana, Vriksasana, Padottanasana, Trikonasana, Adho Mukha Svanasana, Setu Bandha Sarvanghasana, Gomukasana, Vajrasana, Bhadakonasana, Bujangasana, Dandasana, Janu Sirsasana, Paschimottanasana, Setu Bandha Sarvanghasana, Eka Pada Raja Kapotanasana, Supta Bhadakonasana e Savasana. Esses asanas foram escolhidos por terem objetivos e resultados tanto físico quanto psicológico, agindo no bem-estar dos participantes.

Na primeira e na última semana do programa de ioga, os idosos do GE foram submetidos a uma avaliação por meio dos questionários Whoqol-old $\mathrm{e}$ Whoqol-bref, desenvolvidos e validados pela Organização Mundial da Saúde, os quais avaliam os domínios de qualidade de vida (WHO, 2008). O grupo controle (GC) respondeu a ambos os questionários somente por ocasião do término do programa de ioga.

Em 1998, a Organização Mundial da Saúde elaborou um instrumento que conceitua e avalia a qualidade de vida: os questionários Whoqol (World Health Organization Quality of Life). O Whoqol-100 é a versão mais completa desse instrumento. É composto por 100 questões fechadas, que utilizam uma escala de respostas composta por cinco elementos, variando a pontuação entre 1 e 5. A partir do questionário Whoqol-100, foram elaborados mais outros questionários, com perguntas mais específicas, tais 
como Whoqol-old, Whoqol-HIV, dentre outros.

O grupo Whoqol (1996), com a intenção de adaptar o questionário para aplicação em idosos, desenvolveu um instrumento adicional denominado Whoqol-old. Esse questionário deve ser aplicado em conjunto com o Whoqol-bref. O Whoqol-old é composto por apenas um domínio (Envelhecimento) e 24 questões divididas em seis facetas: Habilidades Sensoriais; Autonomia; Atividades Passadas, Presentes e Futuras; Participação Social; Morte e Morrer; e Intimidade (PEDROSO et al., 2011).

Nas questões do questionário Whoqol-old, as escalas variam entre 4 e 20 pontos entre cada faceta, sendo elas Habilidades Sensoriais; Autonomia; Atividades Passadas, Presentes e Futuras; Participação Social; Morte e Morrer; e Intimidade, totalizando uma pontuação entre 24 e 120 pontos.

Sendo a forma abreviada do Whoqol-100, o questionário Whoqol-bref é um instrumento que demanda menor tempo para preenchimento. Composto por duas questões autoavaliativas da qualidade de vida e 24 questões representando cada uma das facetas do Whoqol-100, o Whoqol-bref é formado por quatro domínios: Físico, Psicológico, Relações So- ciais e Meio Ambiente (THE WHOQOL GROUP, 1996).

No questionário Whoqol-bref, a escala de pontos no domínio Físico varia entre 7 e 35 pontos; no domínio Psicológico, entre 6 e 30 pontos; no domínio Relações Sociais, entre 3 e 15 pontos; no domínio Meio Ambiente, entre 8 e 40 pontos. A pontuação total mínima é de 24 pontos e máxima, de 120 pontos.

Para ambos os questionários, os escores das questões são calculados a partir da média aritmética simples. As facetas devem estar todas respondidas numa escala de pontuação entre 1 e 5 pontos. Cada questionário possui 24 questões, logo, a pontuação total mínima será de 24 pontos, e a pontuação total máxima será de 120 pontos.

Tanto no questionário Whoqol-old quanto no questionário Whoqol-bref existem questões que devem ser convertidas na escala de respostas, ou seja, a conversão deve ser utilizada com o objetivo de padronizar todas as respostas dos instrumentos. Portanto, as questões de cada faceta devem ser convertidas para uma mesma escala, na qual o aumento gradativo da resposta equivale, na mesma proporção, ao aumento positivo do resultado da faceta, assim como no Quadro 1.

Quadro 1 - Conversão de questões

\begin{tabular}{|l|c|c|c|c|c|}
\hline \multicolumn{1}{|c|}{ Tipo de escala } & $0 \%$ & $25 \%$ & $50 \%$ & $75 \%$ & $100 \%$ \\
\hline Normal & 1 & 2 & 3 & 4 & 5 \\
Invertido & 5 & 4 & 3 & 2 & 1 \\
\hline
\end{tabular}

Fonte: Pedroso et al. (2011). 
A tabulação e a análise dos dados coletados foram realizadas por meio do pacote estatístico SPSS para Windows na versão 15.0.

O cálculo amostral foi feito com base na equação proposta por Barbetta (2003) para essa finalidade: quando se sabe o tamanho da população. Assumiu-se um erro amostral de 5\%.

\section{Resultados e discussão}

Com relação ao grupo experimental, ao analisar-se a faceta Habilidades Sensoriais, verificou-se que houve aumento da média do pré-teste $(3,51)$ para o pós-teste $(3,85)$, o que significa uma melhora na qualidade de vida para essa variável após a intervenção com ioga. $\mathrm{O}$ resultado do teste $t$ para amostras pareadas foi de $-7,830$, com nível de significância de 0,000 , indicando, portanto, diferença estatisticamente significativa.

Embora tenham sido observados valores de média maiores nos resultados do pós-teste para as demais variáveis analisadas pelo teste $t$ de Student para amostras pareadas, não foram evidenciadas diferenças estatisticamente significativas, conforme a Tabela 1.

Tabela 1 - Resultado do teste $t$ de Student para as variáveis com distribuição normal no pré-teste e no pós-teste $(\mathrm{n}=19)$

\begin{tabular}{l|c|c|c|c}
\hline \multicolumn{1}{|c|}{ Variável } & Média & $\begin{array}{c}\text { Desvio } \\
\text { padrão }\end{array}$ & $\mathrm{t}$ & Sig. \\
\hline Whoqol-old Sensório Pré & 3,5132 & 0,62067 & & 0,000 \\
Whoqol-old Sensório Pós & 3,8553 & 0,62537 & $-7,83$ & 0,272 \\
Whoqol-old Autonomia Pré & 3,6053 & 0,56713 & $-1,134$ & \\
Whoqol-old Autonomia Pós & 3,8158 & 0,49890 & & 0,586 \\
Whoqol-bref Físico Pré & 3,5868 & 0,61079 & & \\
Whoqol-bref Físico Pós & 3,6989 & 0,59309 & $-0,554$ & \\
Whoqol-bref Ambiente Pré & 3,5284 & 0,69562 & & 0,554 \\
Whoqol-bref Ambiente Pós & 3,6542 & 0,52356 & $-0,603$ & \\
\hline
\end{tabular}

Fonte: do autor.

Todas as facetas analisadas pelo teste não paramétrico de Wilcoxon também não apresentaram diferenças estatisticamente significativas, mesmo tendo todas elas (exceto a faceta Morte) apresentado médias do pós-teste ligeiramente supe- riores às médias do pré-teste, conforme a Tabela 2. Dito de outro modo, não se observou piora em nenhum dos indicadores de qualidade de vida na amostra submetida à prática da ioga. 
Para analisar se houve diferenças entre as facetas no grupo de praticantes de ioga (resultados do pós-teste), comparado a um grupo de sedentários que preencheu os questionários Whoqol-old e Whoqol-bref por ocasião do término da intervenção com ioga, foram adotados testes de comparação entre médias para amostras independentes.

Tabela 2 - Resultado do teste de Wilcoxon para as variáveis com distribuição diferente da normal no pré-teste e no pós-teste $(\mathrm{n}=19)$

\begin{tabular}{|c|c|c|c|c|}
\hline Variável & Média & Desvio padrão & Z & Sig. \\
\hline Whoqol-old APPF Pré & 3,87 & 0,75733 & & 0,344 \\
\hline Whoqol-old APPF Pós & 4,0526 & 0,56260 & $-0,946$ & \\
\hline Whoqol-old Social Pré & 3,92 & $\begin{array}{l}0,62799 \\
0,48741\end{array}$ & -02 & 0,358 \\
\hline Whoqol-old Social Pós & 4,0921 & & & \\
\hline Whoqol-old Morte Pré & 3,51 & $\begin{array}{l}0,99864 \\
0,94300\end{array}$ & -0.101 & 0,920 \\
\hline Whoqol-old Morte Pós & 3,4211 & & & \\
\hline Whoqol-old Intimidade Pré & 3,66 & 1,10840 & & 0,732 \\
\hline Whoqol-old Intimidade Pós & 3,8947 & 1,02169 & $-0,343$ & \\
\hline Whoqol-bref Psicológico Pré & 3,64 & $\begin{array}{l}0,63458 \\
0,50922\end{array}$ & -0.967 & 0,333 \\
\hline Whoqol-bref Psicológico Pós & 3,7711 & & & \\
\hline Whoqol-bref Social Pré & 3,7596 & 0,53176 & & 0,597 \\
\hline Whoqol-bref Social Pós & 3,7537 & 0,46882 & $-0,0<0$ & \\
\hline
\end{tabular}

Fonte: do autor.

Foram encontradas diferenças estatisticamente significativas nas facetas Participação Social (teste $t$ de Student); Atividades Passadas, Presentes e Futuras; e Intimidade (teste Mann-Whitney) do questionário Whoqol-old. Apesar de não mostrar diferenças estatisticamente significativas nas outras facetas e domínios de ambos os questionários, todos os resultados (exceto na faceta Habilidades Sensoriais) foram ligeiramente superiores no grupo de idosos que praticaram ioga.

Observando os resultados encontrados no pré-teste realizado com o grupo experimental, verificou-se que os indicadores de qualidade de vida desses idosos já eram favoráveis, o que pode explicar o 
pequeno aumento das mesmas variáveis no pós-teste. Vale ressaltar que Canoas, local em que foi realizada a pesquisa, é uma cidade que investe em políticas públicas voltadas à população idosa, sendo o primeiro município a criar uma Unidade de Pronto Atendimento (UPA) para os idosos, bem como a ter uma Delegacia do Idoso no Rio Grande do Sul. Dando suporte a esses dados, no ano de 2015, Canoas foi selecionada, em concurso nacional de experiências exitosas em saúde do idoso, como uma das dez melhores cidades do país nesse quesito (ROESE, 2014). De acordo com notícias da Prefeitura de Canoas, todos os anos são realizados Jogos de Integração dos Idosos, além de várias outras ações, tais como tai chi chuan, alongamento, caminhada, jogos adaptados, ginástica, dança e academias para a terceira idade com orientação de profissionais adequados. Essas atividades ocorrem durante todo ano (SECOM, 2015).

Com relação às políticas públicas, Benedetti, Gonçalves e Mota (2007) apresentaram e discutiram oito programas de atividade física para idosos (Florianópolis, Brasil; Porto e Coimbra, Portugal; Oleiros e região da Galícia, Espanha; Terranuova Bracciolini, Itália; e Maisach, Alemanha). A coleta de dados foi realizada por meio de entrevista, observação de campo e material de divulgação de cada programa. Os programas de atividade física desenvolvidos em Oleiros, na região da Galícia, e em Terranuova incluíam, entre outras modalidades, a prática da ioga. Interrogados sobre os programas, os idosos praticantes das diversas modalidades (incluindo a ioga) se mostraram agradecidos pela oportunidade de praticar atividades físicas, enfatizando melhora da saúde, aumento do círculo de amizade e maiores oportunidades de lazer. Os autores ainda ressaltaram que a implementação de programas de atividade física para idosos nos diferentes municípios se apresenta como tendência em avanço nas políticas sociais que irão desencadear novos costumes e estilos de vida. Embora considerem que muitas vezes, por trás dessas propostas, estão implícitos interesses econômicos, como a redução de gastos com internações hospitalares e recursos humanos, os ganhos para os idosos repercutem na melhoria da qualidade de vida em qualquer um de seus aspectos (físico, psicológico ou social).

Ferraz e Peixoto (1997) investigaram, por meio de um questionário contendo questões abertas, a qualidade de vida de idosos em uma instituição pública de recreação para essa população na região metropolitana de Belo Horizonte, Minas Gerais. A amostra foi composta por 18 respondentes (12 do sexo feminino e 6 do masculino) com idades entre 60 e 79 anos. Os indicadores de qualidade de vida foram: religião, profissão/ emprego, habitação, sono e repouso, independência, lazer/atividade física, mental e intelectual, saúde e assistência à saúde, dependência de drogas, álcool e fumo, sistema de apoio/convívio social e familiar, descrição positiva do casamento, aspiração/realização e percepção de felicidade. Especificamente, com relação aos indicadores de lazer/atividade física, 
mental e intelectual, na amostra investigada por Ferraz e Peixoto (1997), as três principais atividades de lazer desenvolvidas foram viagens (88\%), dança $(61 \%)$ e jogos (38\%). Embora a ioga não tenha ficado entre as três atividades mais praticadas, ela constava como possível resposta do questionário e foi apontada por alguns respondentes. Esses autores destacaram a importância de o homem se organizar para os anos da velhice quando ainda está em plena atividade, a fim de reduzir o impacto causado pela inatividade. Embora o instrumento de coleta de dados utilizado na pesquisa conduzida por Ferraz e Peixoto (1997) tenha sido diferente do utilizado no presente estudo, os resultados também apontam para uma influência positiva na qualidade de vida de idosos que praticam atividade física. Mais ainda, os autores também encontraram, na instituição investigada, alternativas de manutenção do convívio social e de envolvimento em atividades físicas, recreativas e ocupacionais que justificavam uma percepção de autorrealização, felicidade e bem-estar mais significativos na avaliação da qualidade de vida, como foi o caso do presente estudo.

Baptista e Dantas (2002) conduziram um estudo com o objetivo de investigar as diversas causas e efeitos variados do stress nos aspectos mental, emocional e físico do ser humano, visto que o stress é relevante na redução da saúde e da qualidade de vida. Esses autores defendem que a prática de atividade física complementada com a prática da ioga proporcionam, de um modo mais eficaz, melhora da saúde e da qualidade de vida em função da melhora do estado cognitivo, do equilíbrio emocional, da redução da excitabilidade ou da apatia psicofísica, da melhora da assertividade e da afetividade, do autocontrole da ansiedade e da redução dos sintomas físicos e emocionais do stress. Mais recentemente, Alves, Baptista e Dantas (2006) e Baptista e Dantas (2006) avaliaram os efeitos da prática da ioga sobre flexibilidade, força muscular isométrica submáxima e equilíbrio bem como índices de autonomia funcional em 30 idosas sedentárias submetidas a um programa de ioga, o qual incluía exercícios respiratórios (pránáyámas), de alongamento, equilíbrio e força isométrica submáxima (ásanas) associados ao relaxamento (yoganidra) e à meditação (dhyana), conduzido durante 12 semanas, com três sessões semanais de 60 minutos. $O$ protocolo de coleta de dados foi aplicado nas situações de pré e pós-intervenção e incluiu os testes de caminhar 10min o mais rápido possível (SPILÄ et al., 1996), levantar da posição sentada (GURALNIK et al., 1995; 2000), levantar da posição decúbito ventral (ALEXANDER et al., 1997), o teste Time $U p \& G o$, para avaliar a mobilidade (PODSIADLO; RICHARDSON, 1991), e o teste de comportamento neural do indivíduo (OKUMIYA et al., 1996). Os resultados obtidos do teste $t$ de Student para amostras pareadas indicaram que as melhorias apresentadas em todos os aspectos foram significativas, o que levou os autores a concluir que a ioga pode ser considerada uma prática física alternativa que promove a saúde e a 
qualidade de vida em idosos. Embora os meios de obtenção de evidências também tenham sido diferentes dos utilizados nesta investigação, entende-se que os resultados obtidos por Batista e Dantas (2002), Alves, Baptista e Dantas (2006) e Baptista e Dantas (2006) reforçam as evidências sobre os benefícios da prática da ioga por idosos no sentido de melhoria ou, no caso do presente estudo, de manutenção da qualidade de vida.

\section{Considerações finais}

Neste estudo foi verificado o efeito de um programa de ioga na qualidade de vida de idosos. Após o término do programa de ioga, o grupo experimental apresentou diferença estatisticamente significativa somente na faceta Habilidades Sensoriais. Além disso, também foi comparada a qualidade de vida do grupo de idosos no momento do término do programa de ioga à de um grupo de idosos sedentários. Nesse caso, houve diferenças estatisticamente significativas nas facetas Participação Social, Atividades Passadas, Presentes e Futuras e Intimidade. Apesar de as outras facetas não terem apresentado diferenças estatisticamente significativas, houve um pequeno aumento nas variáveis analisadas no que diz respeito à indicação de melhor qualidade de vida no grupo experimental, exceto nas facetas Habilidades Sensoriais e Morte e Morrer. A partir dos resultados encontrados, embora as diferenças tenham sido pequenas, foram, na sua maioria, favoráveis ao grupo ex- perimental, dando suporte à prática da ioga como fatores que influenciam positivamente na qualidade de vida. Cabe destacar que não houve efeito deletério oriundo da prática da ioga na amostra investigada em nenhuma das facetas analisadas. A título de recomendação, sugere-se que também sejam investigadas variáveis relativas à aptidão física de idosos praticantes de ioga, a fim de melhor compreender outros efeitos dessa modalidade.

\section{The effect of yoga practice in quality of life of elderly}

\section{Abstract}

The aging is a natural and physiological phenomenon of living beings, often associated to negative points, such as degeneration, atrophy, limitations and pain. The yoga therapy, which is a method that addresses the yoga as a form of therapy that aids in recovery and maintenance of health, has been used to improve the quality of life of elders, minimizing the negative factors of aging. This study, characterized as transversal, makes up a final sample of 44 elderly between 65 and 80 years, divided into experimental group and control group. The elderly answered the Whoqol-old and Whoqol-bref questionnaires to assess quality of life and the experimental group underwent yoga classes twice a week for 10 weeks. Statistically significant differences were shown in facets Sensory Skills; Social Participation; Activities of Past, Present and Future; and Intimacy. Despite other facets not have the same results; there was a brief increase in the analyzed variables.

Keywords: Elderly. Quality of life. Yoga. 


\section{Referências}

ALEXANDER, N. B.; ULBRICH, J.; RAHEJA, A.; CHANNER, D. Rising from the floors in onder adults. Journal of the American Geriatrics Society, v. 45, n. 5, p. 564-569, 1997.

ALVES, A. S.; BAPTISTA, M. R.; DANTAS, E. H. M. Os efeitos da prática do yoga sobre a capacidade física e autonomia funcional e idosas. Fitness \& Performance Journal, v. 5, n. 4, p. 243-249, 2006.

ANDERSON, M. I. P. et al. Saúde e qualidade de vida na terceira idade. Textos Envelhecimentos, Rio de Janeiro (RJ), v. 1, n. 1, jan. 1998.

AQUINI, S. N. O yoga e o idoso: "rumo ao alto da montanha": Os efeitos da prática do yoga de acordo com a percepção dos idosos. 2010. 236f. Dissertação (Mestrado em Educação Física)-Programa de Pós-Graduação em Educação Física, Universidade Federal de Santa Catarina, Florianópolis, 2010.

BAPTISTA, M. R.; DANTAS, E. H. M. Yoga no controle de stress. Fit Perf J, v. 1, n. 1, p. 12-20, jan./fev. 2002.

BAPTISTA, M. R.; DANTAS, E. H. M. O yoga na autonomia funcional na terceira idade. Corpus et Scientia, v. 2, n. 2, p. 39-40, set. 2006.

BARBETTA, P. A. Estatística Aplicada às Ciências Sociais. Florianópolis: UFSC, 2003.

BENEDETTI, T. R. B.; GONÇALVES, L. H. T.; MOTA, J. A. P. S. Uma proposta de política pública de atividade física para idosos. Texto Contexto Enferm, Florianópolis (SC), v. 16 n. 3, p. 387-398, jul./set. 2007.

BRASIL. IBGE. Perfil dos idosos responsáveis pelos domicílios no Brasil. 2000. Disponível em: <http://www.ibge.gov.br/home/ estatistica/populacao/perfilidoso/>. Acesso em: 27 jun. 2017.

DIBENEDETTO, M. et al. Effect of gentle Iyengar Yoga Program on gait in the elderly: an exploratory study. Arch Phys Med Rehabil, v. 86, p. 1830-1837, set. 2005.

FERRAZ, A. F.; PEIXOTO, M. R. B. Qualidade de vida na velhice: estudo em uma instituição pública de recreação para idosos. Rev. Esc. Enf. USP, São Paulo (SP), v. 31, n. 2, p. 316-338, ago. 1997.

FUNDO DE POPULAÇÃO DAS NAÇÕES UNIDAS. A força transformadora do envelhecimento da população. Brasília, 30 set. 2016. Disponível em: <http://www.unfpa.org. br/novo/index.php/noticias/ultimas/1371-a-forca-transformadora-do-envelhecimento-da-populacao>. Acesso em: 26 jun. 2017.

GONÇALVES, L. C. et al. Flexibility, functional autonomy and quality of life in elderly yoga practioners. Archives of Gerontology and Geriatrics, v. 53 p. 158-162, 2010.

GURALNIK, J. M. et al. Lower extremity function and subsequent disability consistency across studies, predictive performance models and value of gait speed alone compared with the short physical performance battery. Journal of Gerontology, v. 55, n. 4, p. M221-M231, 2000.

GURALNIK, J. M. et al. Lower extremity function in persons over the age of 70 years as a predictor of subsequent disability. The New England Journal of Medicine, v. 332, n. 9, p. 556-561, 1995.

IYENGAR, B. K. S. Yoga: the path to the holistic health. London: Dorling Kindersley, 2001.

MANTOVANI, E. P., Atividade física, saúde e envelhecimento. Rev. Faculdade de Educação Física UNICAMP, Campinas (SP), p. 65-72, 2013.

OKUMIYA, K. et al. Effects of exercise on neurobehavial function in community-dwelling older people more than 75 years of age. Journal of the American Geriatrics Society, v. 44 , n. 5 , p. 569-572, 1996.

PEDROSO, B. et al. Validação da sintaxe unificada para o cálculo dos escores dos 
instrumentos Whoqol. Rev. Faculdade de Educação Física da UNICAMP, Campinas (SP), v. 9, n. 1, p. 130-156, jan./abr. 2011.

PESTANA, M. H.; GAGEIRO, J. N. Análise de dados para ciências sociais: a complementaridade do SPSS. Lisboa: Sílabo, 2003.

PODSIADLO, D.; RICHARDSON, S. The timed "Up \& Go": a testo of basic functional mobility for frail elderly persons. Journal of the American Geriatrics Society, v. 39, n. 2, p. 142-148, 1991.

ROESE, L. Lançado programa de atendimento em saúde púbica para idosos. Prefeitura Municipal de Canoas, 27 out. 2014. Disponível em: <http://www.canoas.rs.gov.br/site/ noticia/visualizar/id/119313 $\geq$. Acesso em: 13 abr. 2016.

SECOM - SECRETARIA DE COMUNICAÇÃO. Jogos de Integração movimentam idosos canoenses. Prefeitura Municipal de Canoas, 06 out. 2015. Disponível em: <http:// www.canoas.rs.gov.br/site/noticia/visualizar/ id/121843>. Acesso em: 13 abr. 2016.

SOUZA, P. C.; LISBOA, R. A. P. Aplicação do flex teste em idosos praticantes de um programa de yoga. Rev. Faculdade de Educação Física da UNICAMP, Campinas (SP), v. 6, p. 164-173, jul. 2008.

SPILÄ, S. et al. Effects of strength and endurance training on isometric muscle strength and walking speed in elderly women. Acta Physiologica Scandinavica. v. 156, p. 457-464, 1996.

THE WHOQOL GROUP. WHOQOL-bref: introduction, administration, scoring and generic version of assessment - Field Trial Version. Genebra: WHO, 1996.

WHO - WORLD HEALTH ORGANIZATION. Manual WHOQOL-OLD. 2008. (Versão em português). Disponível em: <http://WWW.ufrgs.br/psiq/ WHOQOL-OLD\%20Manual\%20 POrtugues.pdf>. Acesso em: 11 jun. 2015. 